\title{
A contribuição da Ciência da Informação para a Gestão do Conhecimento: um estudo teórico e prático
}

\author{
Narjara Bárbara Xavier Silva \\ Júlio Afonso Sá de Pinho Neto \\ Universidade Federal da Paraíba - UFPB, Brasil
}

CASE REPORT

\begin{abstract}
Resumo
Objetivo. A presente pesquisa teve como objetivo analisar o processo de gestão do conhecimento na empresa Cerâmica Salema, na perspectiva da Ciência da Informação. Método. Revisão bibliográfica, pesquisa aplicada, exploratória e descritiva, com abordagem quantitativa e qualitativa; pesquisa de campo, cujo instrumento utilizado foi um questionário misto, aplicado com o dirigente do nível estratégico, gestores do nível intermediário e demais colaboradores do nível operacional (setores administrativo e de produção industrial). Resultados. A análise de conteúdo dos dados coletados possibilitou identificar as práticas e lacunas existentes em cada etapa do processo em gestão do conhecimento, com base no modelo de processo em GC representado por Bergeron (2003). Conclusões. Os resultados deste estudo são capazes de subsidiar a elaboração de políticas e estratégias para a implantação de um Programa de Gestão do Conhecimento na empresa Cerâmica Salema.
\end{abstract}

Palavras-chave

Ciência da informação ; Gestão do conhecimento ; Processo ; Cerâmica Salema ; Brasil

\section{The Information Science contribution to Knowledge Management: a theoretical study and practical}

\begin{abstract}
Objective. This research aimed to analyze the process of knowledge management in Salema Ceramics Company in perspective of Information Science. Method. Bibliographic review, applied, exploratory and descriptive research, with quantitative and qualitative approach; field research, whose used instrument was a mixed questionnaire, applied with the leader of the strategic level, the intermediate level managers and other employees at the operational level (administrative and industrial production sectors). Results. The analysis of collected data content enabled us to identify existing practices and gaps in each step of the process in knowledge management, based on process model in GC represented by Bergeron (2003). Conclusions. The results of this study are able to subsidize the elaboration of policies and strategies for the implementation of a Knowledge Management Program in Salema Ceramics Company.
\end{abstract}




\section{Introdução}

A partir de uma pesquisa exploratória, é perceptível o crescimento científico no campo da Gestão do Conhecimento (GC) em diferentes contextos organizacionais e em diversas áreas de conhecimento (BARBOSA, 2013). Nessa concepção, a GC é caracterizada como um campo multidisciplinar, ou seja, aquele composto pela justaposição de duas ou mais disciplinas, com foco na proximidade, em que cada disciplina contribui dentro do seu campo de pesquisa (HOLLAND, 2008).

Na perspectiva da Ciência da Informação $(\mathrm{Cl})$, com base nos dados do Repositório BENANCIB, é evidente um crescimento de comunicações sobre Gestão do Conhecimento apresentadas nos Encontros Nacionais de Pesquisa em Ciência da Informação (ENANCIBs), concentrando-se no Grupo Temático 4 (GT 4) denominado "Gestão da Informação e do Conhecimento" (DUARTE et al., 2015). Com base nos fundamentos epistemológicos da $\mathrm{Cl}$, pode-se afirmar que esse contexto científico está em consonância com a abordagem de Araújo (2014) ao situar a Gestão da Informação e do Conhecimento como uma das correntes teóricas ou subáreas que compõem a Ciência da Informação $(\mathrm{Cl})$.

No entanto, apesar desse crescimento científico, a partir de um levantamento das comunicações no ENANCIB, inscritos no GT 4, observou-se o registro de poucas produções científicas sobre GC no âmbito das organizações do setor industrial da construção civil, pois os estudos estão "concentrados nas Instituições de Ensino Superior (IES) e nas organizações ligadas à área de pesquisa” (DUARTE et al., 2015, p. 13). Ao abordar especificamente esse segmento, compreende-se que o mesmo apresenta uma contribuição significativa no desenvolvimento do Brasil, devido à sua importância social e econômica.

Nesse contexto, a Cerâmica Salema foi a empresa selecionada como campo de pesquisa pela facilidade de contato devido a um networking estabelecido anteriormente à escolha. A Cerâmica Salema é caracterizada como uma organização que atua no ramo da indústria do segmento da construção civil. Fundada em 1981, sua fábrica está localizada no Distrito Salema, no município de Rio Tinto (PB), e seu escritório administrativo está localizado na cidade de João Pessoa (PB). Para a escolha da empresa à investigação científica, partiu-se da constatação de que a mesma não possui gestores/setores diretamente responsáveis pela gestão do conhecimento, como também não possui estudos anteriores de análise do processo de GC - objeto de estudo da pesquisa - em mais de 30 anos de atuação, dificultando, assim, o aproveitamento do conhecimento humano e a criação de novos conhecimentos, vetores essenciais para a produtividade empresarial.

Tais considerações iniciais revelam a necessidade de novas pesquisas na Ciência da Informação, com o objetivo de contribuir e consolidar os estudos da área direcionados a esse tipo de organização. Sendo assim, esta pesquisa teve como objetivo geral analisar como se dá o processo de gestão do conhecimento na empresa Cerâmica Salema, na perspectiva da Ciência da Informação. Como objetivos específicos, buscou-se analisar as etapas que contemplam os fluxos informacionais informais e formais no âmbito organizacional (VALENTIM, 2002), ou seja, buscou-se identificar as práticas de gestão do conhecimento (GC) e as práticas de gestão da informação (GI), respectivamente, identificando ainda o processo de $\mathrm{Gl}$ como parte integrante do processo de GC, segundo a abordagem de Sousa, Dias e Nassif (2011). Para tanto, adotou-se como parâmetro para esta pesquisa o modelo de processo em gestão do conhecimento representado por Bergeron (2003).

Em suma, esta pesquisa se justifica para a comunidade científica por atender à elaboração de novas abordagens aplicadas à gestão do conhecimento como uma corrente teórica da Ciência da Informação. Para o âmbito mercadológico, este estudo deve contribuir para o desempenho e a competitividade empresarial, possibilitando melhor aproveitamento das oportunidades de negócios e permitindo que a empresa cumpra o seu papel no desenvolvimento social e econômico do país. Para a sociedade, pretende-se contribuir com a oferta de produtos e serviços de qualidade no âmbito das políticas habitacionais e demais programas governamentais.

\section{Ciência da Informação: contextualização epistemológica}

Conforme Silva e Freire (2012), o advento da Ciência da Informação, no século XX, caracterizou-se pela necessidade urgente de propor procedimentos para tratar os problemas relativos à organização e ao registro e difusão da informação (objeto de estudo) das mais diferentes áreas, principalmente após a explosão informacional. Tais demandas foram decorrentes das pesquisas e investigações realizadas durante a II Guerra Mundial (1939-1945), cujos instrumentos e teorias da Época não eram suficientemente adequados. 
Com relação ao desenvolvimento da $\mathrm{Cl}$ como uma área de conhecimento, a partir de uma revisão de literatura, identificam-se em Capurro (2003), Almeida et al. (2007), Cronnin (2008) e Columbié (2010), pelo menos três paradigmas epistemológicos distintos da $\mathrm{Cl}$ - Físico, Cognitivo e Social -, embora considerados interrelacionados e complementares. Nesse viés, Columbié (2010) ressalta que tais paradigmas auxiliam na abordagem epistemológica das pesquisas no campo da $\mathrm{Cl}$, sendo que cada paradigma abarca os conceitos de usuário, informação e recuperação da informação nos diferentes cenários da sociedade.

De acordo com as abordagens dos autores supracitados, o paradigma físico (Século XVI a XVII) ou Tempo de Gerência da Informação (BARRETO, 2007) é identificado como uma das raízes da Cl e teve como foco o estudo estritamente técnico e mensurável da informação e sua representação em diferentes artefatos. Nesse paradigma, duas são as contribuições consideradas de fundamental importância para a área da Ciência da Informação. A primeira é a publicação do clássico ensaio intitulado As We May Think, de Vannervar Bush (1945), no qual o autor identifica os problemas relativos à explosão informacional na comunidade científica e sociedade em geral, e propõe a criação de uma máquina Memex, uma ferramenta mecanizada e cognitiva de armazenamento e recuperação da informação. Este segundo termo foi cunhado por Calvin Moores, em 1951, e é considerada a segunda contribuição desse Tempo para a área da Cl. Entende-se, portanto, que o paradigma físico considera a importância do uso das Tecnologias da Informação e Comunicação (TICs) nos processos de gerenciamento da informação.

Segundo Columbié (2010), a partir da primeira metade do século XX, no cenário caracterizado como "sociedade da informação" (SARACEVIC, 1996), o Paradigma Cognitivo ou Tempo da relação entre a informação e o conhecimento (BARRETO, 2007) passou a valorizar o conhecimento individual e o sujeito ativo no processo de recuperação da informação, a partir dos estudos da teoria dos "estados anômalos do conhecimento", de Nicholas Belkin (1980); da "equação fundamental (ou cognitiva) da Ciência da Informação", proposta por Brookes (1974); da "noção de três mundos" de Popper (1978) e suas representações - objetiva (mundo exterior do sujeito) e subjetiva (mundo interior do sujeito); e do "modelo cognitivo de recuperação da informação", desenvolvido por Peter Ingwersen (1999). Nesse aspecto, as abordagens do paradigma cognitivo estão direcionadas às necessidades de conhecimento dos sujeitos e às suas formas de internalização.

Finalmente, o paradigma social (segunda metade do século XX) ou Tempo do conhecimento interativo (BARRETO, 2007), além de considerar o aspecto comportamental do usuário, proveniente do Paradigma Cognitivo, passou também a valorizar as interações entre o indivíduo/usuário e o ambiente social no qual ele está inserido. Nesse Tempo, os estudos da "análise de domínio" de Hjørland (1995) caracterizaram o que Almeida et al. (2007) e Cronin (2008) definem como uma abordagem sociocognitiva, na qual busca favorecer a "gestão do conhecimento", levando-se em consideração o conhecimento compartilhado por uma comunidade ou grupo" (ALMEIDA et al., 2007, p. 24). Nesse quesito, o paradigma sociocognitivo aborda a importância da promoção do compartilhamento do conhecimento individual direcionado à criação do conhecimento coletivo no ambiente social.

Numa abordagem reflexiva, corroborando a visão de Columbié (2010), já citado anteriormente, Barreto (2007) afirma que a intenção em representar a origem e o desenvolvimento da Ciência da Informação em três Tempos diferentes não é separar as teorias e práticas da área em períodos fechados, mas sim indicar o foco dos estudos numa determinada época, considerando os seus principais problemas a serem solucionados. Porém, com o desenvolvimento da sociedade da informação e o surgimento de seus diferentes problemas complexos, é possível identificar diversas pesquisas que integram os três paradigmas da Ciência da Informação e suas interdisciplinaridades. Com base nesse levantamento, o presente trabalho abrange os três paradigmas da $\mathrm{Cl}$, considerando as diferentes abordagens apresentadas.

\section{Gestão do Conhecimento: conceitos e dualidades na Ciência da Informação}

Em conformidade com a visão apresentada por Barbosa (2008), ao abordar os aspectos históricos da Ciência da Informação, observa-se que autores como Bush (1945), Moores (1951), Belkin (1980), Brookes (1974), Peter (1999) e Hjørland (1995) já consideravam o conhecimento enquanto fenômeno social do ponto de vista econômico e produtivo. Porém, diferente do enfoque dos autores dessa época, que era mais abrangente, outros autores contemporâneos começaram a abordar o conhecimento dentro de contextos organizacionais. Tal conhecimento é referenciado como um ativo intangível necessário à competitividade dos negócios (STEWART, 
1998). Entre eles merecem destaque os conceitos e dualidades abordados por Nonaka e Takeuchi (1997), Valetim (2002), Cianconi (2003) e Sousa, Dias e Nassif (2011).

A partir de uma dimensão epistemológica, e tomando como base a abordagem de Polanyi (1983), os administradores Nonaka e Takeuchi (1997) identificam o conhecimento em duas categorias. São elas: a) o conhecimento explícito (formal, codificado e transmitido através de processos, documentos e fluxogramas); e b) o conhecimento tácito (informal, não codificado e transmitido socialmente através da interação entre as pessoas). Os autores definem ainda, a criação do conhecimento organizacional como a interação entre esses dois tipos de conhecimento, a saber: socialização (conhecimento tácito $\rightarrow$ conhecimento tácito); externalização (conhecimento tácito $\rightarrow$ conhecimento explícito); combinação (conhecimento explícito $\rightarrow$ conhecimento explícito); e a internalização (conhecimento explícito $\rightarrow$ conhecimento tácito). Tal abordagem influenciou o desenvolvimento de outros estudos teóricos e práticos, com destaque para a área da $\mathrm{Cl}$.

Conforme Valentim (2002), ao abordar a importância da "gestão" desses insumos no contexto organizacional, ou seja, do conhecimento tácito e explícito em prol da produtividade, a Gestão da Informação (GI) é definida como um conjunto de estratégias que trabalha essencialmente os fluxos formais (informação que circunda nos níveis formais) da organização, a fim de apoiar o desenvolvimento das atividades e a tomada de decisão. Já a Gestão do Conhecimento (CG) é conceituada como um conjunto de estratégias que trabalha essencialmente os fluxos informais (informação "gerada" no âmbito das relações interpessoais) da organização, a fim de promover a geração de ideias e a solução de problemas.

Na visão de Cianconi (2003), a GI tem como elementos característicos a informação e os processos, cujo foco, na visão estratégica, é o mapeamento e a gestão de competências em informação, e na visão operacional, é o mapeamento de processos e a gestão de conteúdos. Sendo assim, sua metodologia tem como base o conhecimento explícito. Já a GC, tem como elementos característicos, além da informação e os processos, ou seja, a gestão da informação, o conhecimento e as experiências das pessoas envolvidas na organização. Seu foco, na visão operacional, é a formação de comunidades e a construção de relacionamentos através das interações, enquanto que, na visão estratégica, é a aprendizagem organizacional e a inovação em negócios.

Compreende-se, portanto, que os conceitos e dualidades apresentados demonstram as inter-relações existentes entre a GI e GC, pois enquanto a primeira tem como foco o conhecimento explícito, visando à efetividade no trabalho, a segunda está centrada no conhecimento tácito dos indivíduos, visando à aprendizagem e à inovação. Porém, ambas consideram a informação/conhecimento como um ativo de vantagem competitiva. Nesse contexto, Sousa, Dias e Nassif (2011) afirmam que a gestão da informação é parte integrante do processo de gestão do conhecimento, considerando a Ciência da Informação como base estrutural da GC por dedicar-se aos estudos das propriedades gerais e das condições da informação, como também aos processos que possibilitam seu processamento, sua disponibilização e seu uso efetivo.

Entende-se, portanto, tomando como base os estudos de Nonaka e Takeuchi (1997) e os novos estudos realizados por autores da área da Cl, como Valentim (2002), Cianconi (2003) e Sousa Dias e Nassif (2011), que o campo da Gestão do Conhecimento encontra na Ciência da Informação o respaldo necessário para a sua abordagem como disciplina acadêmica e prática organizacional, a partir do entendimento de que a $\mathrm{Cl}$ tanto tem um componente de ciência pura, por meio da análise dos processos de construção, comunicação e uso da informação, quanto um componente de ciência aplicada, ao conceber produtos e serviços que permitam a construção, comunicação, armazenamento e uso dessa informação em diferentes contextos sociais (BORKO, 1968; LE COADIC, 1996).

\section{0 processo de gestão do conhecimento: modelo de Bergeron (2003)}

Na literatura acadêmica, é possível identificar alguns modelos de processo em gestão do conhecimento que contemplam a gestão da informação como um de seus componentes. Porém, num primeiro momento, faz-se necessário o entendimento do conceito de processo. Segundo Berllo (1999 apud ANGELONI, 2010, p. 34), processo é qualquer fenômeno que apresenta uma contínua mudança no tempo, já que não possui um começo e nem um fim definido, constituindo-se de uma sequência dinâmica de eventos, sendo que cada elemento do processo age e é influenciado por todos os outros. Com base nessa definição, pode-se caracterizar processo como um conjunto de etapas/fases que estão integradas em um ciclo contínuo.

De acordo com o Developer Works (2012), a partir da técnica de modelagem, ou seja, o uso de diagramas para documentar o entendimento do processo, este caracterizado como um fluxo de tarefas, é possível criar o que se denomina por modelo de processo, definido como um objeto que "identifica as atividades essenciais do negócio 
que existem dentro de uma organização" (DEVELOPER WORKS, 2012). No âmbito da gestão do conhecimento, busca-se representar as etapas inter-relacionadas que representam os fluxos informacionais, sejam eles formais ou informais.

Ao tomar como referência tais definições conceituais, dentre os diferentes modelos de processo em GC encontrados no referencial teórico (PROBST; RAUB; ROMHARDT, 2002, BUKOWITZ; WILLIAMS, 2002; BERGERON, 2003), o de Bergeron (2003) foi o selecionado como parâmetro para a pesquisa, pois, além de o modelo permitir o mapeamento dos fluxos informais da organização, ou seja, o conhecimento tácito, também possibilita o mapeamento dos fluxos formais (tipos, ambientes e formatos), a saber, o conhecimento explicitado, e dos recursos informacionais (fontes, serviços, produtos e sistemas), permitindo diagnosticar o processo de gestão do conhecimento na empresa Cerâmica Salema, na perspectiva da Ciência da Informação. Veja figura a seguir.

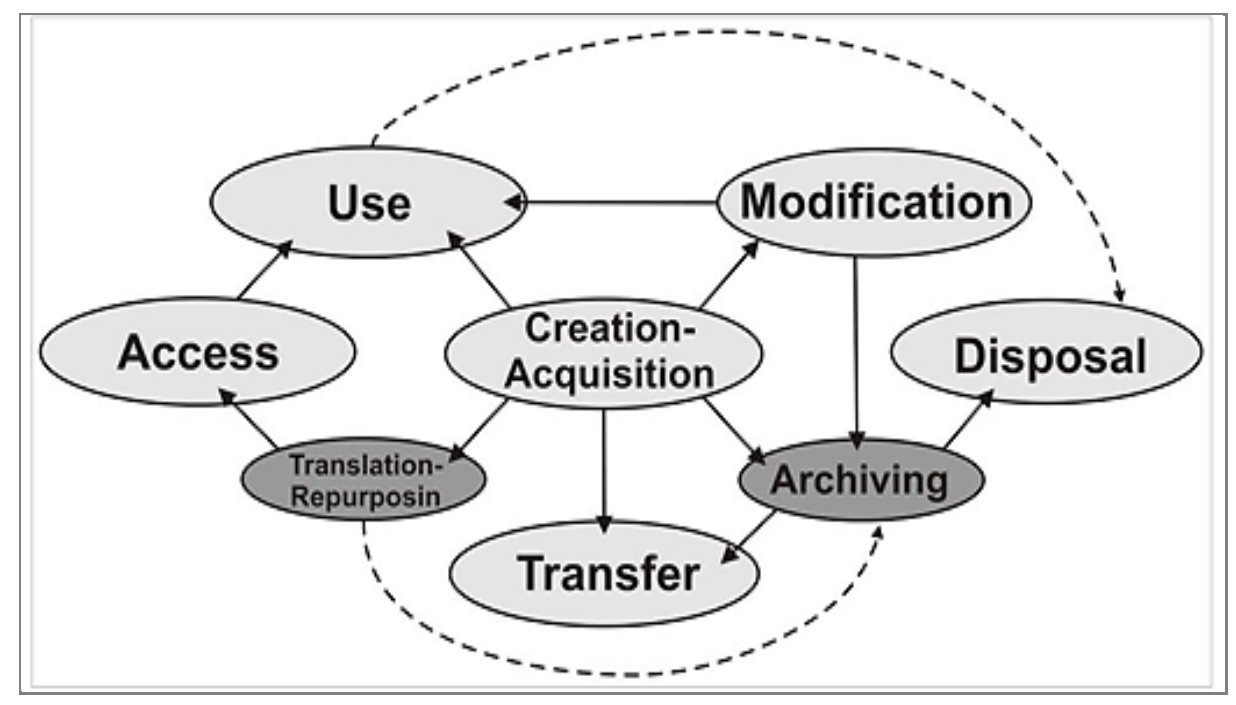

Figura 1 - Process of knowledge management

Fonte: Bergeron (2003, p. 4).

Com base na representação, o processo de gestão do conhecimento organizacional integra oito fases diferentes, são elas:

- Criação ou aquisição (Criation-Acquisition): o conhecimento é criado ou adquirido a partir de fontes externas à organização, considerando os requisitos de conhecimento explícito e tácito necessários ao desempenho organizacional;

- Uso (Use): o conhecimento tácito é utilizado para um fim específico e intencional, considerando as necessidades nas atividades dos membros na organização;

- Arquivamento (Archiving): o conhecimento é explicitado e armazenado sob uma forma e um formato que irá sobreviver no tempo, e será acessível e utilizável para os membros da organização;

- Transferência (Transfer): compartilhamento do conhecimento tácito ou disseminação do conhecimento explícito entre os membros da organização;

- Acesso de utilizadores (Access): provisão de acesso ao conhecimento explícito pelos membros da organização, segundo a sua posição na empresa e as suas necessidades; 
- Modificação (Modification): o conhecimento explícito é editado internamente para suprir necessidades imediatas ou futuras dos membros da organização, considerando os direitos morais do autor original;

- Tradução/reaproveitamento (Translation-Repurposing): o conhecimento explícito adquirido de fontes externas é adaptado da sua forma original para uma forma e linguagem mais apropriadas a uma nova utilização, considerando os direitos morais do autor original;

- Eliminação (Disposal): atualização do conhecimento explícito, identificação e descarte daquele considerado não útil.

\section{Metodologia}

Considerando as características de uma pesquisa, quanto à natureza, o estudo é identificado como sendo aplicado. Quanto aos objetivos, se configura do tipo exploratória e descritiva (GIL, 2010). Já no que se refere à abordagem, optou-se pela quantiqualitativa (MINAYO; SANCHES, 1993).

Com relação ao campo de pesquisa, a Cerâmica Salema é caracterizada como uma empresa de pequeno porte, que atua no setor da indústria da construção civil desde 1981. Quanto à estrutura física, a organização conta com uma fábrica localizada no Distrito Salema, no município de Rio Tinto (PB), e um escritório administrativo localizado na cidade de João Pessoa (PB). Já com relação à estrutura funcional, a Cerâmica Salema conta com dois sócios e 93 colaboradores (total de 95 membros), alocados nos diferentes níveis e respectivos setores organizacionais. Sua missão é "fabricar e vender blocos de cerâmica vermelha para a construção civil", cuja linha de produtos é constituída por uma série de soluções, como os blocos de vedação, alvenaria estrutural, tabelas/blocos para laje, calhas/canaletas, além dos blocos especiais.

Com relação ao objeto de pesquisa - o processo de gestão do conhecimento -, utilizou-se o modelo proposto por Bergeron (2003) por considerá-lo mais adequado ao objetivo do estudo, conforme já mencionado no tópico anterior. O universo ou população da pesquisa equivale ao número de membros alocados nos três níveis organizacionais da empresa estudada, a saber: estratégico, intermediário e operacional (CHIAVENATO, 2003), totalizando 95 sujeitos. O recorte de tais sujeitos pesquisados foi caracterizado como sendo probabilístico por amostragem estratificada não proporcional (GIL, 2010), logo, a amostra é composta por três subgrupos ou subpopulação, são eles: um Diretor Executivo no nível estratégico, quatro gestores no nível intermediário e 21 colaboradores no nível operacional, sendo oito integrantes do setor administrativo e 13 pertencentes ao setor de produção industrial.

Com relação aos procedimentos de coleta dos dados, do ponto de vista de seus aspectos técnicos, o presente estudo se constituiu tendo como base a pesquisa bibliográfica, necessária à fundamentação teórica deste trabalho. A investigação ainda se configurou como uma pesquisa de campo, por se tratar de uma técnica para estudar a realidade de uma empresa, identificando, através de um levantamento de informações junto aos membros da organização, o fenômeno do processo de gestão do conhecimento na perspectiva da Ciência da Informação.

Para tanto, os instrumentos utilizados na coleta de dados, junto aos atores, foram dois tipos de questionário misto (GIL, 2010). O primeiro questionário, direcionado ao Diretor Executivo do nível estratégico da empresa, pretendeu identificar: a) as práticas apoiadas, os meios e as ferramentas disponibilizadas pela organização para promover o processo; b) as diretrizes estabelecidas pela organização para orientar o processo; e c) os setores, equipes ou pessoas responsáveis em gerir os processos de conhecimento no ambiente empresarial. De outro modo, as questões objetivas, aplicadas com os colaboradores do nível intermediário e operacional, pretenderam identificar: a) as práticas realizadas, os meios e as ferramentas utilizadas pelos colaboradores da organização para promover o processo; b) o conhecimento e o cumprimento das diretrizes por estes para orientar o processo; e c) a percepção dos colaboradores quanto à qualidade do processo; além de possibilitar a exposição de sugestões para melhorias ou implantação no processo, a partir das questões abertas.

Finalmente, com relação aos procedimentos de análise dos dados coletados, optou-se pela análise de conteúdo de Bardin (2011), mais especificamente, a análise temática ou categorial. Considerando o modelo de processo 
Categoria I

Práticas de criação/aquisição do conhecimento tácito e explícito externo à organização e sua aplicação imediata nas atividades organizacionais.

Categoria II

Práticas de explicitação do conhecimento tácito, meios de arquivamento e formas de acesso ao conhecimento explicitado.

Categoria III

Práticas, meios e ferramentas de compartilhamento/disseminação do conhecimento tácito/explícito entre os membros da organização.

Categoria IV Existência de conhecimento explícito editado internamento ou adaptado de fontes externas.

Categoria V

Formas de descarte do conhecimento explícito não útil.

em GC de Bergeron (2003), adotado como parâmetro para a pesquisa, delimitou-se as seguintes categorias de análise:

Quadro 1 - Categorias de Análise

Fonte: Elaboração própria, com base em Bergeron (2003).

\section{Análise e discussão dos resultados}

A partir da abordagem de Chiavenato (2003) e das informações levantadas na pesquisa de campo, foram identificados três diferentes perfis de sujeitos na empresa Cerâmica Salema. No nível estratégico, o Diretor Executivo tem 29 anos, possui pós-graduação lato sensu e ocupa o cargo há quatro anos. No nível intermediário, todos os respondentes possuem curso superior completo e têm entre 28 e 37 anos de idade. Com base nos dados, o menor tempo de ocupação no setor é de 14 meses e o maior tempo é de oito anos.

No nível operacional, com relação à subpopulação do setor administrativo, $63 \%$ dos pesquisados possuem curso superior completo, $25 \%$ possuem o ensino médio completo e $13 \%$ possuem o curso superior incompleto. Quanto à faixa etária, os respondentes têm entre 24 e 36 anos de idade. O menor tempo de ocupação no setor é de 18 meses e o maior tempo é de oito anos. Já no que se refere à subpopulação do setor de produção, $69 \%$ dos pesquisados possuem ensino fundamental incompleto, $15 \%$ possuem o ensino fundamental completo e os outros $15 \%$ possuem ensino médio completo. Com relação à faixa etária, os respondentes têm entre 27 e 50 anos de idade. O menor tempo de ocupação no setor é de dois meses e o maior tempo é de 24 anos.

Tomando como referência o enfoque dos instrumentos de pesquisa e as categorias de análise, têm-se os resultados apresentados e analisados a seguir.

\subsection{Práticas, meios e ferramentas para promover o processo}

Na Categoria I, a Cerâmica Salema identifica e seleciona o conhecimento necessário à gestão e inovação em negócios. Nesse sentido, o fluxo das informações ocorre tanto no curso externo para o interno à organização, a partir das diferentes fontes de aquisição do conhecimento, quanto no curso interno para o externo, por meio das soluções sugeridas pelos colaboradores e aplicadas no ambiente empresarial para atender às demandas do mercado. Segundo dados da pesquisa, $60 \%$ dos membros afirmaram propor soluções para os processos produtivos da empresa "muito frequentemente" ou "frequentemente". Conforme entrevista com o gestor, quando aplicadas, tais soluções agregam valor ao negócio "muito frequentemente".

Na Categoria II, a Cerâmica Salema possui uma sistemática de retenção do conhecimento, a partir dos mecanismos disponibilizados para armazenamento do conhecimento explícito, sendo os mais utilizados as unidades físicas, virtuais e os sistemas de informação. Vale destacar que, com base na pesquisa, foi identificado o uso de um portal corporativo, uma plataforma que integra diferentes tecnologias da informação e comunicação, a partir de uma interface única de acesso (FREITAS; QUINTANILLA; NOGUEIRA, 2004, p. 83). 
Nesse sentido, 44\% dos colaboradores, todos alocados nos níveis intermediário e operacional/administrativo, utilizam essa ferramenta "muito frequentemente" ou "frequentemente".

Na Categoria III, a Cerâmica Salema possui práticas para o compartilhamento/disseminação do conhecimento tácito e explícito entre os colaboradores, como as comunidades de prática (48\% de participação), os fóruns de discussão (80\% de participação), o banco de melhores prática e lições aprendidas (24\%), a gestão da memória organizacional (24\%) e o benchmarking (24\%). Outra ação evidenciada foi a "identificação das competências organizacionais e individuais" que, apesar de ser realizada informalmente por $32 \%$ dos trabalhadores, ou seja, não dispõe de registros no repositório de conhecimentos explícitos, a prática é reconhecida pela organização e se encontra em processo de formalização a partir da estruturação de um banco de informações.

Com relação às tecnologias de suporte ao processo de gestão do conhecimento, identifica-se o uso "muito frequente" (12\%) ou "frequente" (28\%) das ferramentas de colaboração entre os membros da organização, além do uso "frequente" dos Sistemas de Gestão de Conteúdo - SGC (20\%) e Sistemas de Apoio à Decisão - SAD (32\%), a fim de promover o gerenciamento efetivo do conhecimento explicitado.

Ainda na Categoria III, a Cerâmica Salema possui diversos veículos para a promoção da comunicação interna, considerando o perfil de cada nível organizacional e as características de cada veículo de comunicação, sendo os mais utilizados o quadro de avisos (48\%), o e-mail (40\%), as palestras (60\%) e as reuniões administrativas $(36 \%)$. Vale destacar que uma ferramenta indicada como sendo de uso "muito frequente" (4\%) ou "frequente" (20\%) ou "esporádico" (28\%) no setor de produção foi a caixa de sugestões, o que comprova o grau de participação desses membros na sugestão de soluções à empresa, já que esse instrumento tem o objetivo de permitir um fluxo ascendente de informações, ou seja, possibilita a comunicação dos colaboradores para a alta administração, a partir da exposição de opiniões, necessidades e sugestões de melhoria.

Na Categoria IV, segundo o dirigente entrevistado, no caso dos documentos armazenados no portal corporativo, por exemplo, existe o acompanhamento das edições por meio da notificação de modificação e identificação de quem modificou ao autor original. Com base nos dados coletados, um número significativo de colaboradores (44\%) consegue identificar o autor original dos documentos editados internamente ou adaptados de fontes externas "muito frequentemente" ou "frequentemente". Enfim, na Categoria $V$, a empresa possui um plano de atualização e backup regular dos documentos, bem como se preocupa com o descarte seguro do conhecimento explícito considerado não útil.

\subsection{Diretrizes para orientar o processo}

Com base na Figura 2 a seguir, na Categoria I, 92\% dos colaboradores da Cerâmica Salema conseguem identificar quais os conhecimentos necessários ao desenvolvimento da empresa, influenciando diretamente no número de ideias sugeridas para a melhoria dos processos e produtos.

Na Categoria II, a organização possui diretrizes que orientam o processo de armazenamento das informações, a partir de planos de documentação e gestão de banco de dados. Tais diretrizes são conhecidas por $66 \%$ dos colaboradores, refletindo, assim, nas contribuições válidas para a memória organizacional, ou seja, no número de arquivamentos por parte dos membros que são aprovados pela organização (64\% de aprovação). Ainda nessa categoria, a Cerâmica Salema possui diretrizes para o acesso seguro à informação, a fim de manter a "confidencialidade" e "disponibilidade" (SÊMOLA, 2006) dos documentos acessados. Tais diretrizes são conhecidas pela maioria dos colaboradores da organização $(72 \%)$, contribuindo para a segurança das informações corporativas. 


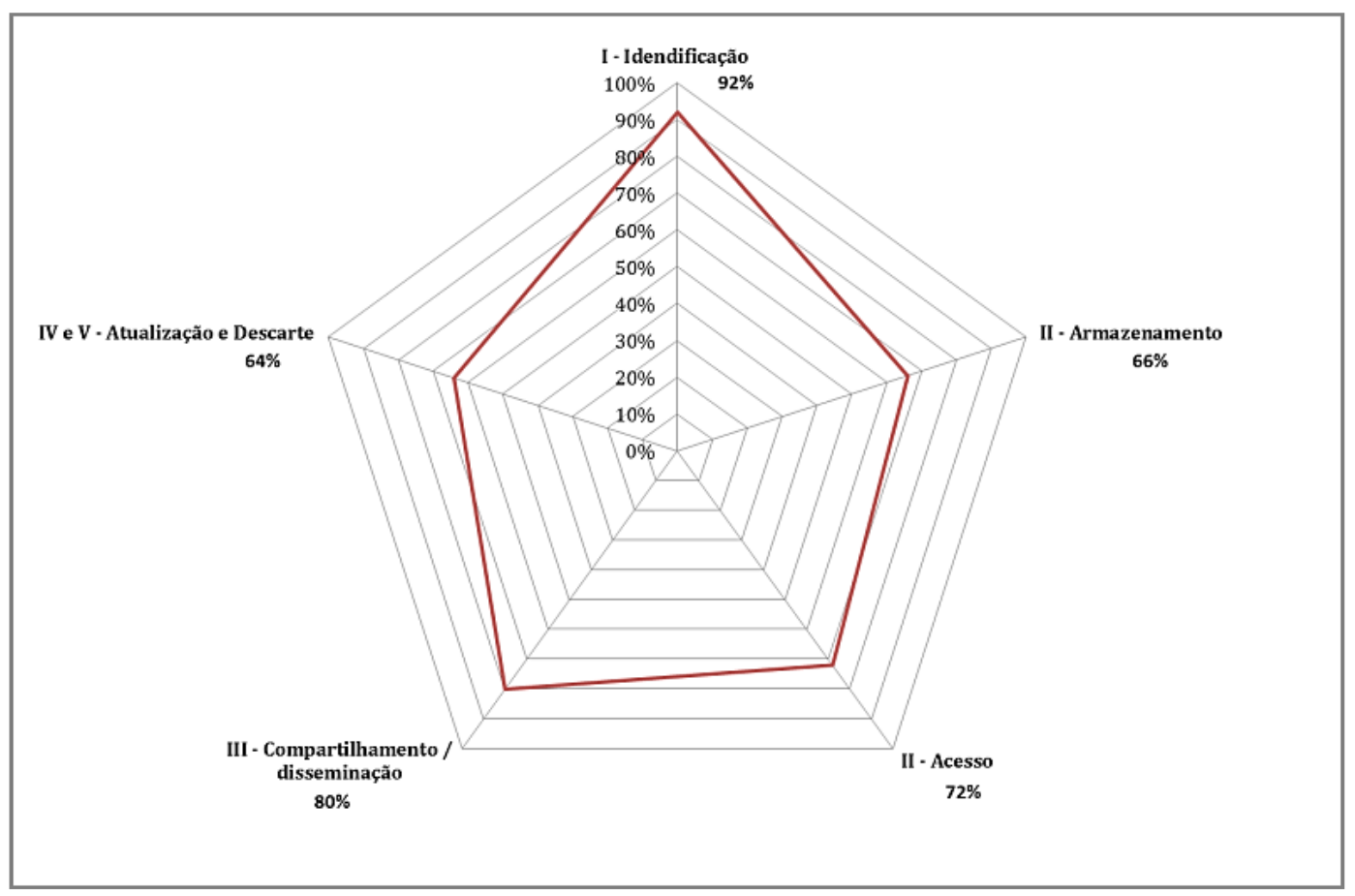

Figura 2 - Diretrizes para orientar o processo

Fonte: Dados da pesquisa (2015).

Na Categoria III, a Cerâmica Salema apresenta diretrizes para a transferência do conhecimento (compartilhamento/disseminação), que são conhecidas por $64 \%$ dos colaboradores. Enfim, na Categoria IV e V, constatou-se que a Cerâmica Salema possui critérios estabelecidos para a edição/adaptação do conhecimento explícito, como também para a atualização de informações (pessoais, empresariais ou dos clientes) e o descarte seguro daquelas consideradas não úteis. Tais diretrizes são conhecidas por $40 \%$ dos membros da empresa, influenciando diretamente na "integridade" (SÊMOLA, 2006) das informações acessadas e utilizadas no ambiente empresarial.

\subsection{Qualidade do processo}

Quanto à qualidade do processo como um todo, segundo o Diretor Executivo da Cerâmica Salema, os setores da empresa realizam funções de gestão de pessoas, gestão da informação, gestão da comunicação, gestão de relacionamento com o cliente, gestão da tecnologia e gestão de segurança da informação, cujas atividades estão relacionadas ao processo de gestão do conhecimento. No entanto, não existe um setor ou pessoa específica para a promoção e o acompanhamento do processo na organização.

A partir da pesquisa de campo, têm-se os seguintes dados com relação à percepção dos colaboradores: 


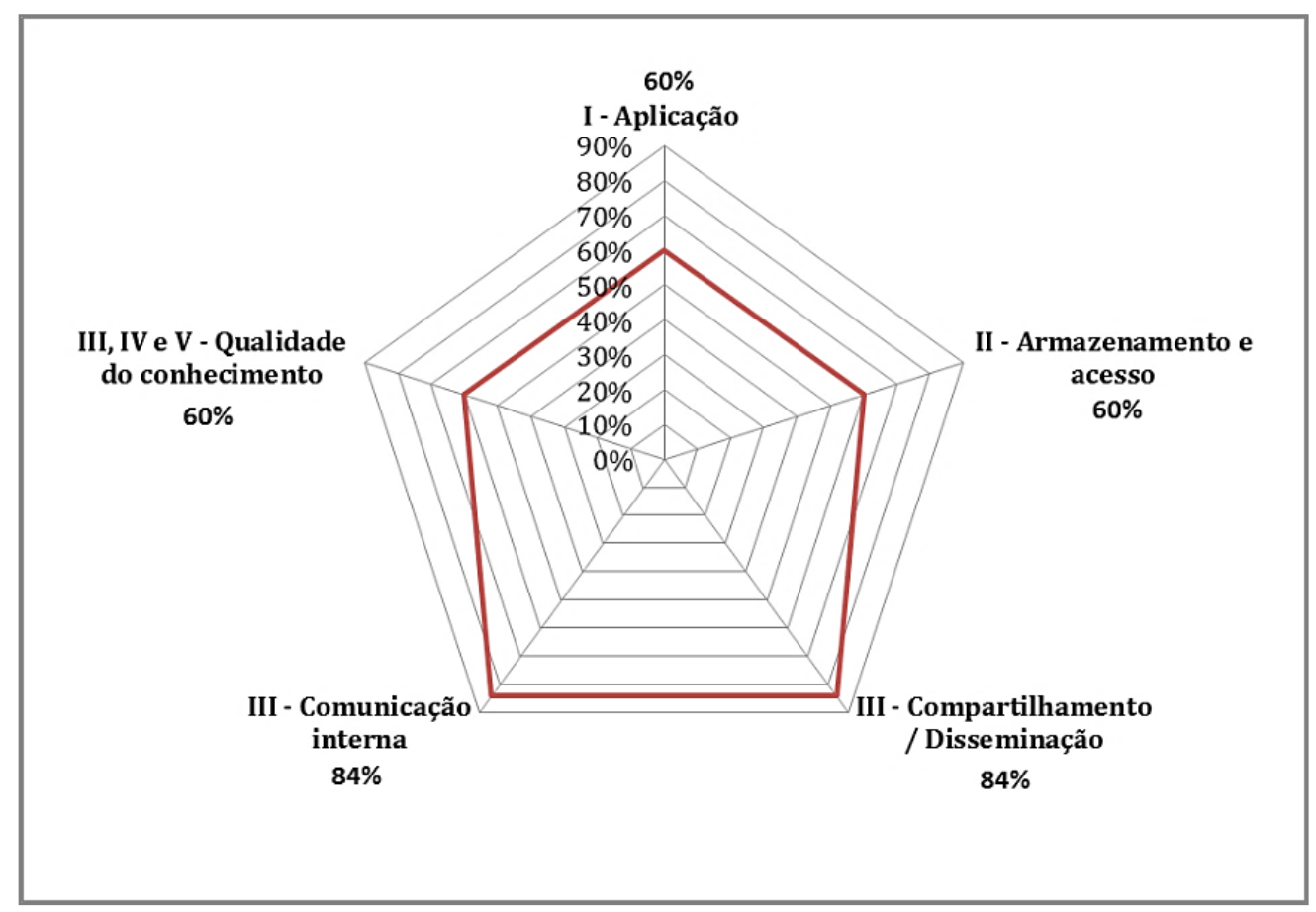

Figura 3 - percepção dos colaboradores quanto à qualidade do processo

Fonte: Dados da pesquisa (2015).

Com base na representação, na Categoria I, 60\% dos membros pesquisados da Cerâmica Salema estão "satisfeitos" quanto à frequência de aplicação das ideias sugeridas por eles para o desenvolvimento da organização, permitindo a participação destes no negócio da organização. Na Categoria II, 4\% dos colaboradores estão "muito satisfeitos" e 56\% "satisfeitos" quanto à efetividade dos mecanismos de armazenamento e acesso às informações corporativas. Na Categoria III, 4\% estão "muito satisfeitos" e $80 \%$ "satisfeitos" quanto à contribuição das práticas de compartilhamento do conhecimento tácito e explícito para o aprendizado individual. Já com relação aos meios de comunicação interna, $84 \%$ dos respondentes estão "satisfeitos" quanto à contribuição dos meios de comunicação interna para se atualizarem sobre o mercado, a empresa e o setor. Enfim, com relação às Categorias $I V$ e $V, 60 \%$ dos funcionários estão "satisfeitos" quanto à qualidade das informações empresariais, pois estas são consideradas compreensíveis, atuais e relevantes para o desempenho individual e organizacional (FELIX, 2003).

\section{Conclusões}

Esse estudo teve como enfoque identificar as práticas, os meios e as ferramentas; as diretrizes; e a qualidade do processo em GC na empresa Cerâmica Salema. Tais elementos têm a função de promover, orientar e otimizar as etapas do processo, respectivamente. Nessa concepção, os objetivos específicos foram discutidos e analisados a partir do referencial teórico e dos dados coletados, cujos resultados podem ser categorizados em práticas de gestão do conhecimento, ou seja, de criação, compartilhamento/disseminação do conhecimento tácito e sua aplicação na inovação dos processos, produtos e/ou serviços; práticas de gestão da informação, a saber, ações de explicitação, arquivamento e disseminação do conhecimento explicitado; e tecnologias da informação e comunicação como suporte aos processos.

Porém, apesar das práticas identificadas, considerando o grau de insatisfação dos sujeitos pesquisados e as opiniões explanadas nos questionários aplicados, foram evidenciadas algumas lacunas nas etapas do processo em GC, com base no modelo de Bergeron (2003). Com relação às práticas de gestão do conhecimento, apesar da ambiência inovadora, caracterizada pela abertura da empresa para receber e experimentar novas ideias, identificou-se que essa abordagem não se estende, de forma satisfatória, ao nível operacional/produção, podendo comprometer o desempenho desse perfil de trabalhadores.

Identificou-se, ainda, que ações como cursos, treinamentos e capacitações não são realizadas de forma contínua e abrangente, o que poderia contribuir, substancialmente, caso ocorressem, para o desenvolvimento 
das competências individuais dos colaboradores, principalmente aqueles situados no nível operacional/produção. Tais competências são necessárias ao exercício das funções em cada setor e promoção da criatividade no ambiente de trabalho, influenciando diretamente na produtividade e competitividade empresarial.

Com relação às práticas de gestão da informação, identificou-se a importância e a necessidade de um banco de competências individuais e organizacionais para a promoção do compartilhamento do conhecimento tácito entre os colaboradores, bem como a melhoria da comunicação interna e disponibilização de meios para a retenção do conhecimento no nível operacional/produção, a partir dos mecanismos já utilizados pela organização.

Já com relação às tecnologias da informação e comunicação como suporte aos processos de GI e GC, percebeu-se a necessidade de maior uso das ferramentas workflow e SGC e das diferentes tecnologias GED, a fim de contribuir com a melhoria da qualidade das informações e com a afetividade dos mecanismos de armazenamento e acesso às informações no ambiente corporativo. Identificou-se, ainda, a necessidade de avaliação da arquitetura da informação no portal corporativo, a fim de identificar se as suas funcionalidades estão coerentes com as necessidades dos colaboradores e se a sua estrutura tecnológica permite o seu uso de forma efetiva. Tal avaliação irá permitir um diagnóstico mais preciso da contribuição dessa plataforma para o processo de gestão do conhecimento na organização.

Finalizando, é importante ressaltar que este estudo é capaz de subsidiar a elaboração de políticas e estratégias para a implantação de um Programa de Gestão do Conhecimento na empresa Cerâmica Salema, a partir da avaliação das iniciativas já implementadas e a identificação das lacunas em cada etapa do processo.

\section{Referências} ALMEIDA, Daniela et al. Paradigmas contemporâneos da Ciência da Informação: a recuperação da informação como ponto
focal. Revista Eletrônica Informação e Cognição, v. 6, n. 1, p. 16-27, 2007. Disponível em: $<$ http://www.portalppgci.marilia.unesp.br/reic>. Acesso em: 04 ago. 2011.

ANGELONI, Maria Terezinha. Comunicação nas organizações da Era do Conhecimento. São Paulo: Atlas, 2010.

ARAÚJO, Carlos Alberto Ávila. Fundamentos da Ciência da Informação: correntes teóricas e o conceito de informação. Perspectivas em Gestão \& Conhecimento, João Pessoa, v. 4, n. 1, p. 57-79, jan./jun. 2014.

BARBOSA, Ricardo Rodrigues. Gestão do Conhecimento na literatura acadêmica: um estudo sobre a produção científica na base Scopus. In: ENCONTRO NACIONAL DE PESQUISA EM CIÊNCIA DA INFORMAÇÃO, 13., 2013. Florianópolis. Anais... Florianópolis: ANCIB, 2013

BARDIN, L. Análise de conteúdo. Lisboa: Edições 70, 2011.

BARRETO, Aldo de Albuquerque. Uma história da Ciência da Informação. In: TOUTAIN, Lídia Maria Batista Brandão (Org.). Para entender a Ciência da Informação. Salvador: EDUFBA, 2007.

BERGERON, B. P. Essentials of knowledge management. Chichester: John Wiley \& Sons, 2003.

BORKO, H. Information science: what is it? American Documentation, v. 19, n. 1, p. 3-5, jan. 1968.

BUKOWITZ, W.; WILLIAMS, R. Manual de gestão do conhecimento: ferramentas e técnicas que criam valor para a empresa. Porto Alegre: Bookman, 2002.

BUSH, V. As we may think. Atlantic Magazine, July 1945. Disponível em: <http://www.ps.uni-sb.de/ duchier/pub/vbush/vbushall.shtml>. Acesso em: 18 out. 2012.

CAPURRO, Rafael. Epistemologia e ciência da informação. In: ENCONTRO NACIONAL DE PESQUISA EM CIÊNCIA DA INFORMAÇÃO, 5. 2003, Belo Horizonte. Anais... Belo Horizonte: ANCIB, 2003.

CERÂMICA SALEMA. Disponível em: <http://ceramicasalema.com.br/>. Acesso em: 05 jan. 2015.

CHIAVENATO, Idalberto. Introdução à teoria geral da administração: uma visão abrangente da moderna administração das organizações. 7. ed. rev. e atual. Rio de Janeiro: Elsevier, 2003. 
CIANCONI, Regina de Barros. Gestão do conhecimento: visão de indivíduos e organizações no Brasil. Tese (Doutorado) Escola de Comunicação, Universidade Federal do Rio de Janeiro, Rio de Janeiro, 2003. Disponível em: <http://www.uff.br/ppgci/editais/ tese\%20regina\%20cianconi.pdf>. Acesso em: 22 nov. 2013.

CRONIN, B. The sociological turn in Information Science. Journal of Information Science, v. 34, n. 4, p. 465-475, 2008.

DEVELOPER WORKS. Modelagem de processos em negócio e seus impactos positivos nas organizações. Disponível em: https://www.ibm.com/developerworks/community/blogs/fd26864d-cb41-49cf-b719-

d89c6b072893/entry/modelagem de processos de neg C3 B3cio bpm e seus impactos positivos nas organiza C3 A7 C3 B5es1?lang=en. Acesso em: 28 nov. 2014.

DUARTE, Emeide Nóbrega et al. Práxis de Gestão do Conhecimento no ambiente das organizações no escopo da Ciência da Informação. In: ENCONTRO NACIONAL DE PESQUISA EM CIÊNCIA DA INFORMAÇÃO, 16, 2015. João Pessoa. Anais... João Pessoa: ANCIB, 2015.

FELIX, W. Introdução à gestão da informação. Campinas: Alínea, 2003.

FREITAS, Rogério Afonso de; QUINTANILLA, Leslie Wittig; NOGUEIRA, Ari dos Santos. Portais Corporativos: uma ferramenta estratégica para a gestão do conhecimento. Rio de Janeiro: Brapsport, 2004.

GIL, A. C. Como preparar projetos de pesquisa. 5. ed. São Paulo: Atlas, 2010.

HOLLAND, G. A. Information Science: an interdisciplinary effort? Journal of Documentation, v. 64, n. 3, p. 7-23, 2008.

LINARES COLUMBIÉ, R. Epistemología y ciência de la información: repensando um diálogo incluso. Acimed, Havana. v. 21, n. 2, 2010. Disponível em: <http://www.acimed.sld.cu/index.php/acimed/article/viewArticle/52/18>. Acesso em: 22 out. 2012.

LE COADIC, Y. F. A Ciência da Informação. Brasília: Briquet de Lemos, 1996.

MINAYO, M. C. S; SANCHES, O. Métodos qualitativos e quantitativos: oposição ou complementaridade? Caderno de Saúde Pública, Rio de Janeiro, v. 9 n. 3, p. 239-262, jul./set., 1993. Disponível em: <http://www.scielo.br/scielo.php?pid=S0102311X1993000300002\&script=sci arttext>. Acesso em: 01 maio 2014.

NONAKA, Ikujiro; TAKEUCHI, Hirotaka. Criação de conhecimento na empresa: como as empresas japonesas geram a dinâmica da inovação. 12. ed. Rio de Janeiro: Campus, 1997

POLANYI, M. The tacit dimension. Gloucester: Peter Smith, 1983.

PROBST, G.; RAUB, S.; ROMHARDT, K. Gestão do conhecimento: os elementos construtivos do sucesso. Porto Alegre: Bookman, 2002.

SÊMOLA, Marcos. Gestão da segurança da informação. In: STAREC, Cláudio; GOMES, Elizabeth; BEZERRA, Jorge (Org.). Gestão estratégica da informação e inteligência competitiva. São Paulo: Saraiva, 2006.

SILVA, J. L. C.; FREIRE, G. H. A. Um olhar sobre a origem da ciência da informação: indícios embrionários para sua caracterização identitária. Encontros Bibli, v. 17, n. 33, p. 1-29, 2012. Disponível em:

<http://www. periodicos.ufsc.br/index.php/eb/article/view/1518-2924.2012v17n33p1/21708>. Acesso em: 20 out. 2012.

SOUSA, Edvânio Duarte de; DIAS, Eduardo José Wense; NASSIF, Mônica Erichsen. Gestão da informação e gestão do conhecimento na Ciência da Informação: perspectivas teóricas e práticas organizacionais. Informação \& Sociedade: Estudos, João Pessoa, v. 21, n. 1, p. 55-70, jan./abr. 2011. Disponível em: <http://www.ies.ufpb.br/ojs/index.php/ies/article/view/4039>. Acesso em: 26 nov. 2013

VALENTIM, M. L. P. Inteligência competitiva em organizações: dado, informação e conhecimento. DataGramaZero, v. 3, n. 4, p. 1-13, ago. 2002. Disponível em: <http://www.dgz.org.br/ago02/Art 02.htm>. Acesso em: 01 nov. 2013. 


\section{Dados dos autores}

\section{Narjara Bárbara Xavier Silva}

Possui graduação em Comunicação Social - habilitação em Relações Públicas, pela Universidade Federal da Paraíba (UFPB). Mestrado em Ciência da Informação, pela Universidade Federal da Paraíba (PPGCI - UFPB). Atuou como Estagiária Docente do Departamento de Comunicação e Turismo da UFPB, como Tutora EAD do curso de extensão em Educação em Direitos Humanos (UFPB Virtual) e como Pesquisadora/Instrutora do Projeto de Inclusão Digital no âmbito do Programa Rede Digital (FapesqPB). Desde 2013 é membro do Grupo de Pesquisa em Informação, Aprendizagem e Conhecimento (GIACO | PPGCI - UFPB) e em novembro de 2014 começou a atuar como Agente Local de Inovação (ALI) no Programa Agentes Locais de Inovação na Paraíba (Programa ALI-PB | SEBRAE/CNPq).

narjara.barbara@gmail.com

\section{Júlio Afonso Sá de Pinho Neto}

Possui graduação em Comunicação Social, Habilitação Relações Públicas, pela Universidade Federal do Maranhão (1984), mestrado em Comunicação e Cultura pela Universidade Federal do Rio de Janeiro (1995), doutorado em Comunicação e Cultura pela Universidade Federal do Rio de Janeiro (1999) e Pós-Doutorado em Ciência da Informação pela Faculdade de Comunicação e Documentação da Universidade de Murcia (Espanha), (2012). Atualmente é professor associado do Departamento de Comunicação da Universidade Federal da Paraíba, coordenador do Curso de Relações Publicas e professor do Curso de Mestrado e Doutorado do Programa de Pós-Graduação em Ciência da Informação - PPGCI da UFPB. Coordena o projeto de pesquisa A inclusão digital para deficientes visuais no Setor Braille da Biblioteca Central da UFPB (Edital 14/2011 CNPq), o Projeto Ações de inclusão digital nas instituições penitenciárias do Brasil e Espanha: um estudo comparado (Edital 07/2011 CNPq/CAPES) e o Projeto de Extensão Inclusão Digital para Agentes de Limpeza e Agentes Ambientais da Coleta Seletiva do Lixo da cidade de João Pessoa/PB (Fapesq). É líder do Grupo de Pesquisa Tecnologias da Informação e Comunicação.

sadepinho@uol.com.br

Recibido - Received: 2016-01-27

Aceitado - Accepted: 2016-03-02

\section{$(\mathrm{cc}) \mathrm{EY}^{\mathrm{B}}$}

This work is licensed under a Creative Commons Attribution 4.0 United States License.

\section{UILIS D-Sole}

This journal is published by the University Library System of the University of Pittsburgh as part of its D-Scribe Digital Publishing Program and is cosponsored by the University of Pittsburgh Press. 